\title{
Spatial Resolution and the Magnitude of Infarct Volume Measurement Error in DWI in Acute Ischemic Stroke
}

\author{
(D).M. Ospel, (D) A. Jaffray, (D) V. Schulze-Zachau, (D). Kozerke, and (D) C. Federau
}

\begin{abstract}
BACKGROUND AND PURPOSE: Infarct volume in acute ischemic stroke is an important prognostic marker and determines endovascular treatment decisions. This study evaluates the magnitude and potential clinical impact of the error related to partial volume effects in infarct volume measurement on diffusion-weighted MR imaging in acute stroke and explores how increasing spatial resolution could reduce this error.
\end{abstract}

MATERIALS AND METHODS: Diffusion-weighted imaging of 393 patients with acute stroke, of whom 56 had anterior circulation large-vessel occlusion, was coregistered to standard space. Lesion boundaries were manually segmented. A 3D lesion-volume model was resampled for voxel sizes from $4 \times 4 \times 8$ to $1 \times 1 \times 2 \mathrm{~mm}$, and the surface-volume, corresponding to the partial volume error, was calculated. The number of cases with anterior circulation large-vessel occlusion, in which the endovascular therapy core threshold of $70 \mathrm{~mL}$ was contained within the margin of error, was calculated as a function of imaging resolution.

RESULTS: The mean infarct core volume was $27.2 \pm 49.9 \mathrm{~mL}$. The mean surface volume was $14.7 \pm 20.8 \mathrm{~mL}$ for $2 \times 2 \times 4 \mathrm{~mm}$ resolution and $7.4 \pm 10.7 \mathrm{~mL}$ for $1 \times 1 \times 2 \mathrm{~mm}$ resolution. With a resolution of $2 \times 2 \times 4 \mathrm{~mm}, 70 \mathrm{~mL}$ was contained within the margin of error in $7 / 56$ cases (12.5\%) with large-vessel occlusion, while with a $1 \times 1 \times 2 \mathrm{~mm}$ voxel size, the margin of error was $3 / 56(5 \%)$. The lesion-volume range of potentially misclassified lesions dropped from $46.5-94.1 \mathrm{~mL}$ for a $2 \times 2 \times 4$ mm resolution to $64.4-$ $80.1 \mathrm{~mL}$ for a $1 \times 1 \times 2 \mathrm{~mm}$ resolution.

CONCLUSIONS: Partial volume effect is an important source of error in infarct volume measurement in acute stroke. Increasing spatial resolution substantially decreases the mean error. Standard use of high-resolution DWI should be considered to increase the reliability of infarct volume measurements.

ABBREVIATION: LVO = large-vessel occlusion

nfarct core volume is a known predictor of outcome in patients with acute stroke; smaller infarct cores are associated with better outcomes and higher rates of functional independence. In patients with a small infarct core, thrombectomy leads to a more favorable result. ${ }^{1-4}$ Diffusion-weighted imaging is considered the

Received December 11, 2019; accepted after revision March 6, 2020. From the Department of Radiology (J.M.O., V.S.-Z., C.F.), University Hospital Basel, University of Basel, Basel, Switzerland; Department of Clinical Neurosciences (J.M.O.), University of Calgary, Calgary, Canada; and Institute for Biomedical Engineering (A.J., S.K., C.F.), University of Zurich, Zurich, Switzerland.

This work was supported, in part, by the Swiss Heart Association.

Preliminary results of this study previously presented at: Annual Meeting of the American Society of Neuroradiology, May 18-23, 2019; Boston, Massachusetts.

Please address correspondence to Christian Federau, MD, Institute for Biomedical Engineering, University of Zurich, Gloriastr 35, 8092 Zürich, Switzerland;

e-mail: christian.federau@gmail.com; @johanna_ospel

Indicates article with supplemental on-line appendix.

Indicates article with supplemental on-line photos.

http://dx.doi.org/10.3174/ajnr.A6520 criterion standard for infarct core measurement. A large infarct core, usually above $70 \mathrm{~mL}$, was considered a contraindication for endovascular therapy in many clinical trials and is commonly used as a threshold in clinical practice. ${ }^{5-7}$

The accuracy of DWI-based infarct core measurement is limited by several factors. For example, although DWI hyperintense lesions are generally considered to represent irreversibly damaged tissue, reversible DWI lesions have been reported. ${ }^{8,9}$ Another important potential source of error is partial volume effects at the border of the lesion due to the finite spatial resolution reconstruction.

The surface volume (ie, the volume of the surface voxels) represents the volume in which misclassification can occur due to the partial volume effect and is an established measure of spatial resolution-induced measurement error. ${ }^{10}$ This error is equal to the number of voxels composing the surface, multiplied by the individual voxel volume used in image reconstruction (Fig 1). Because of the complex shapes of most large stroke lesions (eg, those with a gyriform pattern), volume measurement error due 
to partial volume effects could potentially lead to an erroneous choice of therapy.

The purpose of our study was to assess the magnitude and potential therapeutic impact of errors related to partial volume effects in infarct core volume measurement on DWI in acute stroke. Furthermore, we aimed to evaluate to what extent increasing the spatial resolution could reduce this error.

\section{MATERIALS AND METHODS}

\section{Patients}

Approval from the local ethics committee was obtained for this anonymized, retrospective evaluation. MR imaging examinations of patients who presented in the emergency department of the University Hospital of Basel with symptoms of acute ischemic stroke between August 2010 and November 2017 were reviewed and considered for this study. A subgroup analysis was performed in the group with anterior circulation large-vessel occlusion (LVO) who be considered endovascular treatment-eligible according to

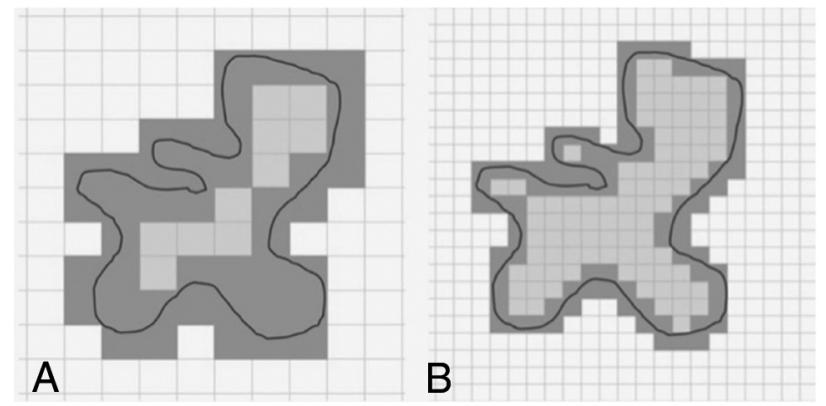

FIG 1. Inaccuracy of volumetric measurements at the lesion surface. The grid represents voxel boundaries; the solid line represents an acute ischemic lesion. Voxels outlined in gray are the surface voxels, ie, those voxels with lesion margins. The sum of the surface voxel volume is a measure of the potential measurement error due to partial volume effects, which results from discretization at the lesion surface (the net volume uncertainty per surface voxel has been defined as half of the volume of the voxel in both the positive and negative directions). Standard resolution leads to large surface volume and thus has the potential for large measurement errors $(A)$. Increasing the spatial resolution substantially reduces the surface volume and thereby decreases the potential measurement error $(B)$. their occlusion site based on current American Heart Association/ American Stroke Association guidelines. ${ }^{11}$

\section{Technical Parameters}

MR imaging examinations were performed on a Magnetom Skyra 3T, a Magnetom Skyra fit 3T, or a Magnetom Avanto fit 1.5T scanner (Siemens) and included diffusion-weighted (acquired at $b=1000 \mathrm{~s} / \mathrm{mm}^{2}$ ), fluid-attenuated inversion recovery, and susceptibility-weighted images. The Table provides a summary of the acquisition parameters.

\section{Image Postprocessing}

All diffusion-weighted images were coregistered to the standard Montreal Neurological Institute template (http://www.bic.mni. mcgill.ca/ServicesAtlases/ICBM152NLin2009) space rotated in the anterior/posterior commissure direction, using an affine transformation and a mutual information loss, and resampled to a standard resolution of $128 \times 128 \times 40$ voxels with the Advanced Normalization Tools library (http://stnava.github.io/ ANTs/). Images were then saved back in the DICOM format using a homemade $\mathrm{C}++$ code.

\section{Infarct Core Segmentation}

Infarct cores, if present, were manually segmented by 2 radiologists (J.M.O., V.S.-Z.) on the coregistered $b=1000 \mathrm{~s} / \mathrm{mm}^{2}$ DWI using an open-source DICOM viewing software (horosproject.org). Tissue was identified as infarct on the basis of hyperintensity compared with the surrounding tissue and the contralateral hemisphere. Imaging artifacts resembling infarct cores were distinguished from real infarct cores on the basis of contextual information from the whole image including symmetry/bilaterality, presence of metals, other causative materials, and typical anatomic location near the skull base or on brain-bone/air interfaces. Parenchymal hematomas in continuation with or within infarcts were included in the segmentation. In case of doubt, to distinguish hyperintensity due to acute infarction from leukoaraiosis and chronic white matter changes, we reviewed the other available MR images, including the $b=0 \mathrm{~s} / \mathrm{mm}^{2}$ images and fluid-attenuated inversion recovery images.

\section{Diffusion-weighted imaging acquisition parameters}

\begin{tabular}{lccc}
\hline \multicolumn{1}{c}{ Parameter } & Mean & SD & Range \\
\hline Section thickness (mm) & 3.03 & 0.22 & $3-5$ \\
TR (ms) & 6989.76 & 1518.53 & 3.32 \\
TE (ms) & 99.59 & - & $3550-9600$ \\
Interslice gap (mm) & 0.6 & 168 & $59-104$ \\
Sequence duration (sec) & 583 & & $0.6-0.6$ \\
Field strength & $1.5 T: n=251(63.9 \%)$ & $312-984$ \\
& $3 T: n=142(36.1 \%)$ & \\
Matrix size & $128: n=282(71.8 \%)$ & \\
& $162: n=106(27.0 \%)$ & \\
In-plane resolution & $192: n=5(1.3 \%)$ & \\
& $2.00 \times 2.00 \mathrm{~mm}: n=318(81.0 \%)$ & \\
& $1.36 \times 1.36 \mathrm{~mm}: n=46(11.7 \%)$ & \\
& $1.20 \times 1.20 \mathrm{~mm}: n=4(1.0 \%)$ & \\
& $1.13 \times 1.13 \mathrm{~mm}: n=1(0.3 \%)$ & \\
\end{tabular}

Note:-- indicates that there was no SD as the interslice gap was $0.6 \mathrm{~mm}$ in all cases. 


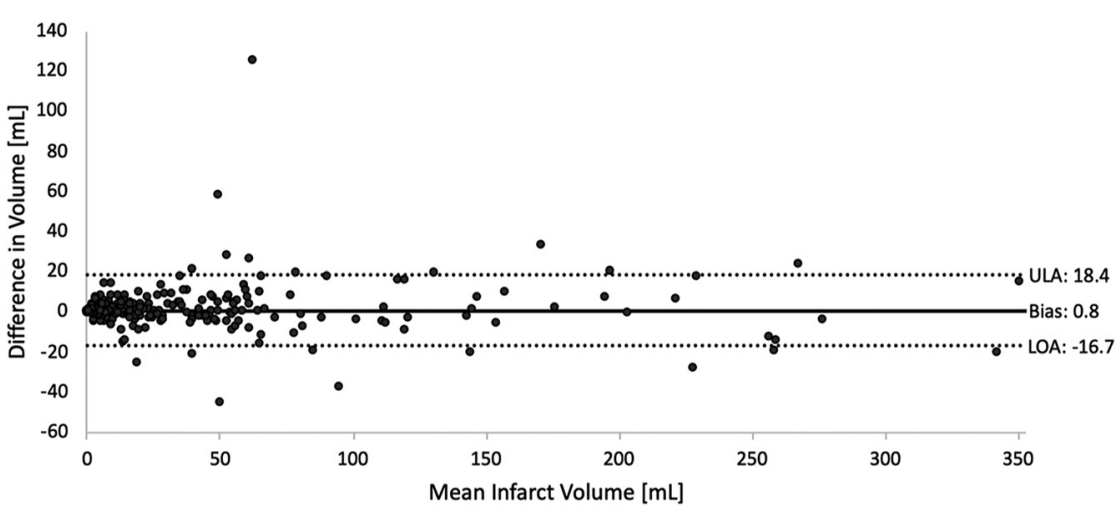

FIG 2. Interreader agreement of manual infarct core segmentation. Bias $(0.82 \mathrm{~mL})$ is shown with a solid line. Lower limits of agreement (LOA, $-16.74 \mathrm{~mL})$ and upper limits of agreements (ULA, $18.38 \mathrm{~mL}$ ) are marked with dashed lines.

\section{Lesion Tessellation}

Section-by-section parameterization of segmented ischemic cores using spline interpolants was performed to reconstruct lesion boundaries with arbitrarily high in-plane resolution, and tessellated surfaces were reconstructed from the interpolated lesion boundary points, thereby providing lesion boundaries of varying in-plane resolutions. Reconstruction of the high-resolution 3D lesions with voxels of fixed size within the tessellated surface boundaries was then performed for a continuous range of voxel sizes from $4 \times 4 \times 8$ to $1 \times 1 \times 2 \mathrm{~mm}$ using a Matlab routine (MathWorks). Section-by-section parameterization and lesion tessellation are illustrated in detail in the On-line Appendix.

\section{Data Analysis and Statistics}

Differences in the segmented stroke volumes between the 2 radiologists were visualized with a Bland-Altman plot. Infarct core volumes and infarct core surface volumes were calculated for both readers and were averaged for further analysis. Means and SDs were calculated for averaged stroke volume and surface volume, and the number of patients in whom the margin of error (ie, mean infarct volume $\pm 50 \%$ of the mean surface volume) contained $70 \mathrm{~mL}$, which is the commonly used decision threshold for endovascular treatment, ${ }^{5,6}$ was calculated for each voxel size. A Spearman correlation was performed to assess the relationship of spatial resolution and surface volume. Correlation coefficients and corresponding $P$ values were calculated for each patient and averaged.

\section{RESULTS}

\section{Data Base Characteristics}

MR imaging examinations of 393 patients with DWI-hyperintense lesions consistent with acute infarction were included in this study (220 male patients, $56.0 \%$ ). The mean age was $71.6 \pm 14.2$ years. Fifty-six patients had an anterior circulation large-vessel occlusion (26 male patients, $46.4 \%$; mean age, $71.7 \pm 14.9$ years; median NIHSS score, 8 ; interquartile range, 12-17.5), with a median time from symptom onset to MR imaging of 26 hours (interquartile range, 21.8-30.8 hours). These included $34 \mathrm{M} 1$ segment middle cerebral artery occlusions, 21 terminal internal carotid artery occlusions, and 1 A1 segment anterior cerebral artery occlusion.

\section{Human Segmentation}

Comparison of segmentation volumes performed by both radiologists showed good agreement: In 380/393 (96.03\%), the difference was within the limits of agreement (Fig 2). Mean infarct volume was $27.2 \pm 49.9 \mathrm{~mL}$, and $69.4 \pm 81.1 \mathrm{~mL}$ in the subgroup of patients with anterior circulation LVO. Thirty-two of 393 (8.1\%) infarct volumes were above $70 \mathrm{~mL}$. In $8 / 393$ (2.0\%) infarct volumes, the readers disagreed on whether the infarct volume was greater or less than $70 \mathrm{~mL}$. On-line Fig 3 shows how human segmentations were performed on an exemplary case.

\section{Volume Error Related to Partial Volume Effects}

The mean surface volume (ie, volume error related to partial volume effects) was $14.7 \pm 20.8 \mathrm{~mL}$ for a reconstruction voxel size of $2 \times$ $2 \times 4 \mathrm{~mm}$ and $7.4 \pm 10.7 \mathrm{~mL}$ for a size of $1 \times 1 \times 2 \mathrm{~mm}$. In the patients with anterior circulation LVO, the mean surface volume was $64.8 \pm 31.1 \mathrm{~mL}$ for a reconstruction voxel size of $2 \times 2 \times$ $4 \mathrm{~mm}$ and $17.7 \pm 16.3 \mathrm{~mL}$ for a size of $1 \times 1 \times 2 \mathrm{~mm}$. Figure 3 shows the simulation results for additional spatial resolution steps. There was a strong and highly significant correlation between spatial resolution and surface volume $(P=0.903, P<.001)$.

\section{Volume Range Containing the Clinical-Decision Volume Threshold of $70 \mathrm{~mL}$ as Function of Reconstruction Resolution}

With a reconstruction voxel size of $2 \times 2 \times 4 \mathrm{~mm}$, the clinical-decision volume threshold of $70 \mathrm{~mL}$ was contained in a lesion volume ranging from 46.5 to $94.1 \mathrm{~mL}$ - that is, lesions as small as $46.5 \mathrm{~mL}$ could have been erroneously classified as $>70 \mathrm{~mL}$ due to partial volume effects, and lesions of $94.1 \mathrm{~mL}$ volume could have been erroneously classified as $<70 \mathrm{~mL}$ (Fig $4 B$ ). Reducing the reconstruction voxel size to $1 \times 1 \times 2 \mathrm{~mm}$ resulted to the clinical-decision volume threshold being contained in the range of 64.4-80.1 mL (Fig 4C).

\section{Number of Patients with Anterior Circulation LVO within the Range of Measurement Error}

In the patients with anterior circulation LVO, the clinical-decision volume threshold of $70 \mathrm{~mL}$ was contained within the range of measurement error in 7/56 cases (12.5\%) with a resolution of $4 \times 2 \times$ $2 \mathrm{~mm}$ and in $3 / 56$ cases (5.3\%) with a resolution of $1 \times 1 \times 2 \mathrm{~mm}$ (Fig 5).

\section{DISCUSSION}

Accurate determination of infarct volume in acute ischemic stroke is crucial because infarct core volume was used as an exclusion criterion in several randomized controlled trials ${ }^{6,11}$ and has become a key decision driver in endovascular therapy decision- 

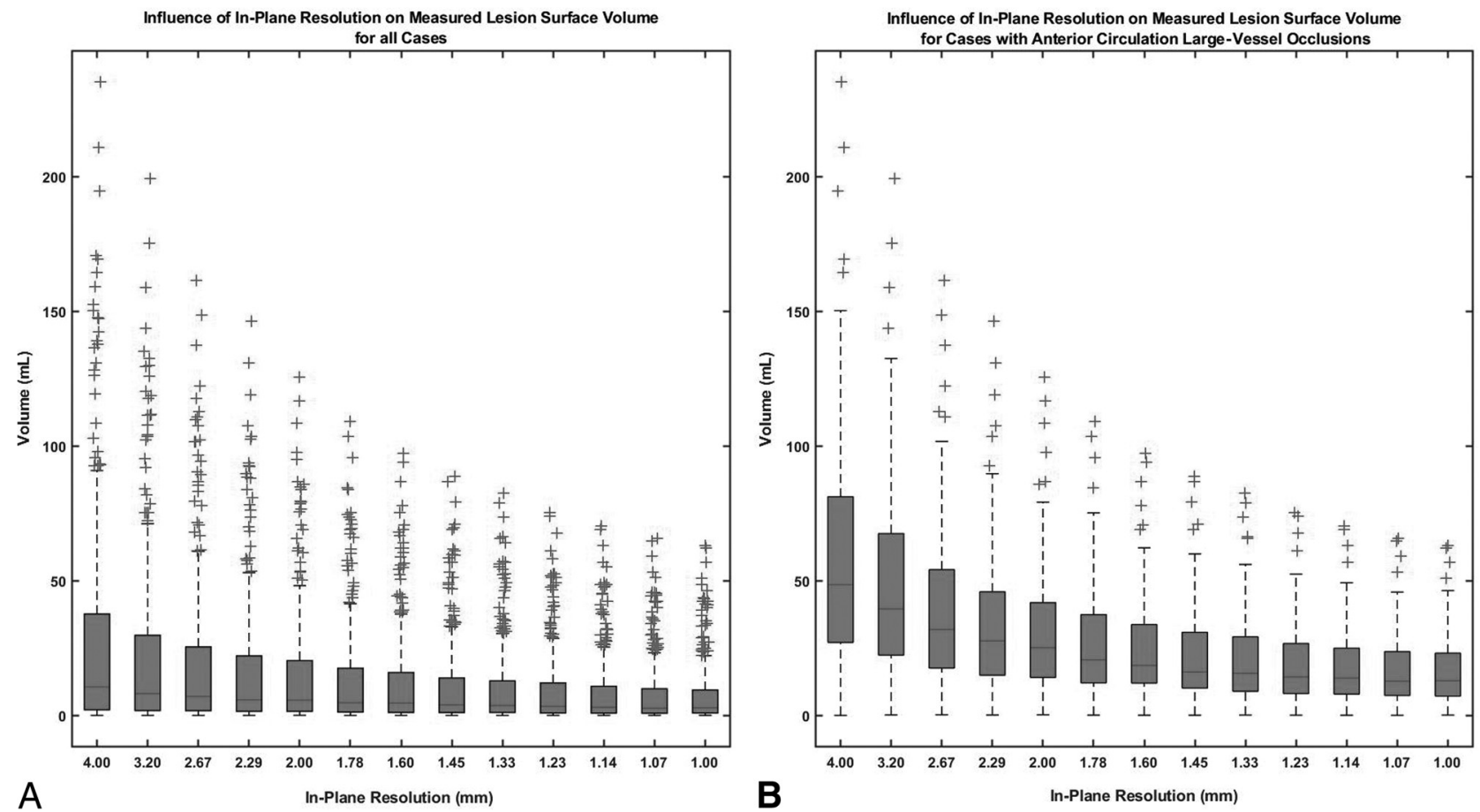

FIG 3. Surface volume as a function of spatial resolution for all cases $(A)$ and for the cases with anterior circulation large-vessel occlusion $(B)$. Surface volume (median and interquartile range) are shown on the $y$-axis, and spatial resolution, on the $x$-axis. With increasing spatial resolution, a marked decrease in surface volume can be observed.
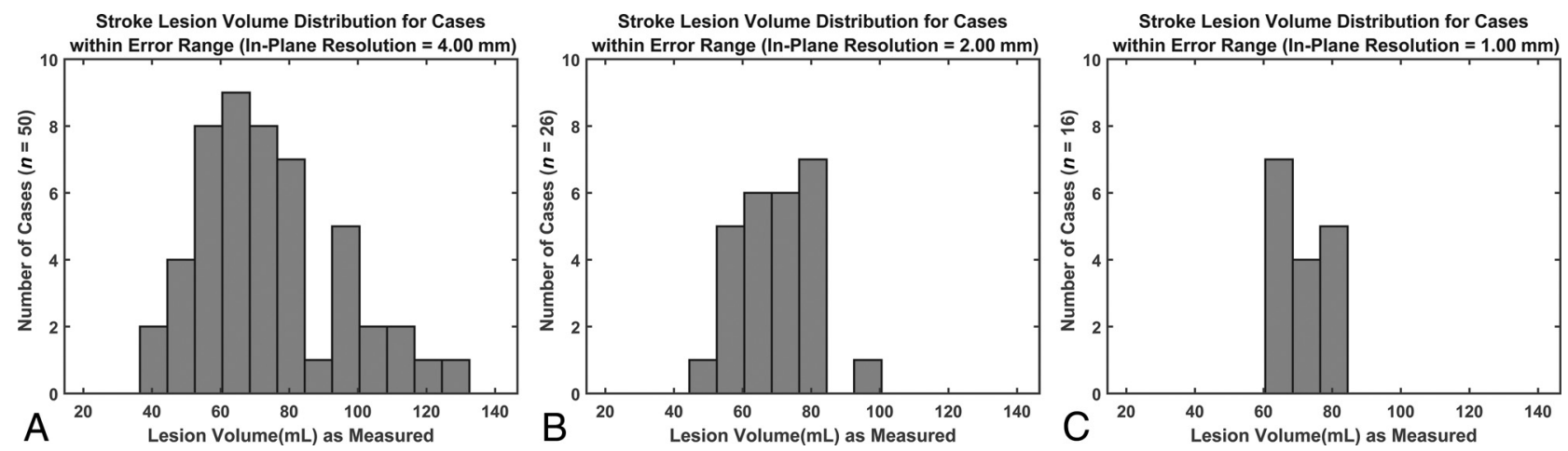

FIG 4. Distribution of stroke lesion volumes for the cases in which $70 \mathrm{~mL}$ is contained within the margin of measurement error for voxel sizes of $4 \times$ $4 \times 8 \mathrm{~mm}(A), 2 \times 2 \times 4 \mathrm{~mm}(B)$, and $1 \times 1 \times 2 \mathrm{~mm}(C)$. With increasing spatial resolution, the range of lesion volumes for which the margin of error contains $70 \mathrm{~mL}$ is reduced. Lesion ranges were as follows: $4 \times 4 \times 8 \mathrm{~mm}(A)$ : $40.96-126.46 \mathrm{~mL}$; $2 \times 2 \times 4 \mathrm{~mm}(B): 46.5-94.1 \mathrm{~mL}$; and $1 \times 1 \times 2 \mathrm{~mm}(C)$ : $64.35-80.13 \mathrm{~mL}$.

making. Our study demonstrates the importance of spatial resolution when performing DWI-based infarct measurements in the context of acute stroke imaging: With a reconstruction resolution of $2 \times 2 \times 4 \mathrm{~mm}$, the mean error in our patient cohort was $14.7 \mathrm{~mL}$, and a resolution of $1 \times 1 \times 2 \mathrm{~mm}$ led to a noteworthy decrease of the error to $7.4 \mathrm{~mL}$. The same held true for the number of cases in which $70 \mathrm{~mL}$ was contained within the margin of error in volume measurements (from $12.5 \%$ of cases with LVO using a $2 \times 2 \times 4 \mathrm{~mm}$ resolution to $5 \%$ of cases using a $1 \times$ $1 \times 2 \mathrm{~mm}$ resolution), while the range of lesion volumes measured in these cases decreased from 46.5-94.1 to 64.4-80.1 mL.

Our findings suggest that DWI in the context of acute stroke should be acquired with the maximal resolution possible. Of note, the maximal achievable resolution is variable across scanners and depends mostly on the imaging gradient strength. While the increase in resolution implies a decrease in the signal-to-noise ratio, this should be acceptable because of the high contrast of the DWI findings positive for lesions compared with the normal brain parenchyma. In case the signal-to-noise ratio needs to be improved, scan time could be increased. ${ }^{12}$ However, stroke outcomes are highly time-dependent, and treatment delays should, therefore, be avoided. If a high-resolution DWI sequence were to be used in the acute setting, its acquisition time has to be relatively short. High-resolution DWI sequences with acquisition times of $<5$ minutes are available and have been shown to increase lesion conspicuity and the overall number of visible lesions in acute ischemic stroke. ${ }^{13,14}$ 


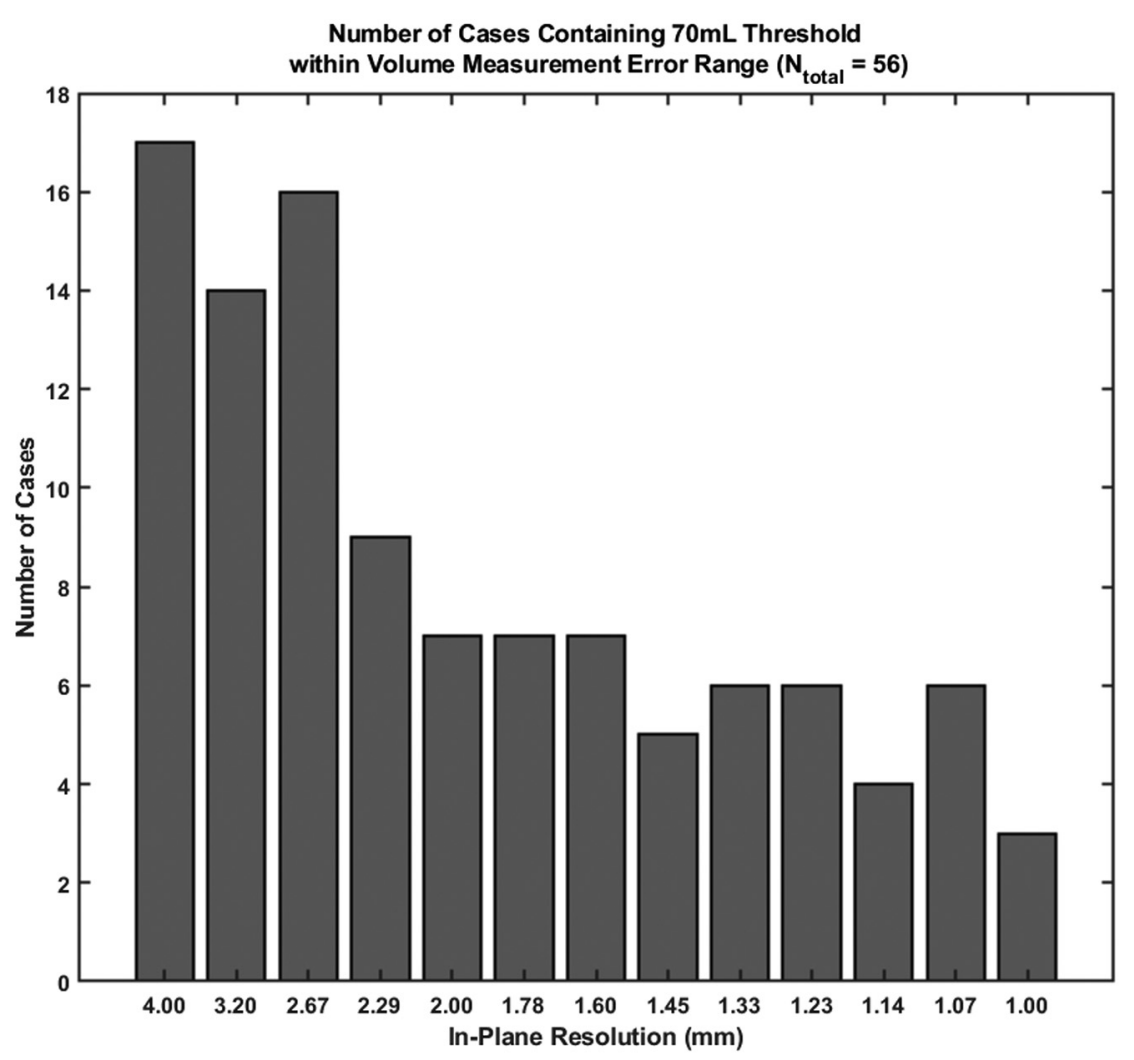

FIG 5. The number of cases in which $70 \mathrm{~mL}$ is contained within the margin of error ( $y$-axis) for the cases with anterior circulation large-vessel occlusion, ie, cases in which a treatment error could have resulted on the basis of a $70-\mathrm{mL}$ threshold, as a function of spatial resolution (x-axis). With increasing spatial resolution, the number of cases in which the margin of error contains $70 \mathrm{~mL}$ is reduced. increased motion artifacts due to longer required scan times. Furthermore, endovascular therapy trials that used DWI for infarct core measurement and established the 70-mL threshold might have had the very same measurement inaccuracy described in this article but were nevertheless able to show the clinical significance of this threshold. DWI volume is used in most multicentric studies as a surrogate marker for stroke severity, for example in the Diffusion Weighted Imaging Evaluation for Understanding Stroke Evolution Study 2 (DEFUSE 2) studies, ${ }^{17}$ but in clinical practice, pretreatment MR imaging is performed only in a limited number of centers. Despite these limitations, our study provides preliminary exploratory evidence for the magnitude of infarct core measurement error on DWI that can arise due to partial volume averaging and offers a starting point for future investigations on how to reduce these inaccuracies.

\section{CONCLUSIONS}

When one performs DWI-based volumetric stroke infarct measurements with routinely used spatial resolutions, measurement errors due to partial vol-

Several further sources of errors that can occur in DWI-based infarct core measurement were not considered in this work, such as inhomogeneities in the $\mathrm{B}_{0}$ and diffusion-weighted gradient and imaging gradient fields, ${ }^{2,3}$ errors in section selection, and motion artifacts. ${ }^{15,16}$ Because the effect of these errors is multiplicative, our results most probably substantially underestimated the real volume measurement error. Reducing measurement error due to partial volume averaging is even more important in patients with larger, complex-shaped infarcts (ie, gyriform, white matter-sparing infarct patterns) because the lesion surface and hence the potential error due to partial volume averaging are particularly large in such patients.

\section{Limitations}

Our study has several limitations: First, this was a single-center study. The images used were mostly acquired after thrombolytic or endovascular therapy, but it is reasonable to assume that the sizes and shapes of the lesions were similar to lesions in the therapeutic window. Infarct segmentation was performed manually, possibly introducing some inaccuracy, though interreader agreement of the infarct segmentations was good. We performed a "virtual increase" using lesion tessellation to simulate high-resolution DWI, and not "real" high-resolution DWI, which has been shown to be feasible at and beyond the smallest voxel size simulated in this study but yields its own set of challenges that have not been addressed in this study. These include a decrease in signal-to-noise ratio with voxel size and ume averaging are substantial and can potentially affect clinical decision-making. Thus, infarct volumes derived from DWI should be interpreted with caution. The magnitude of error can be minimized when spatial resolution is increased. Because fast and robust high-resolution DWI sequences are available, they should be used in acute stroke imaging whenever possible to accurately measure infarct cores and come to a solid, well-informed endovascular therapy decision.

Disclosures: Johanna M. Ospel—RELATED: Grant: University of Basel Research Foundation, Julia Bangerter-Rhyner Foundation, Freiwillige Akademische Gesellschaft Basel, Comments: research scholarships. Christian Federau—RELATED: Grant: Swiss National Science Foundation.* Victor Schulze-Zachau-UNRELATED: Employment: employment as resident physician at the University Hospital Basel.* Money paid to institution.

\section{REFERENCES}

1. Ozdemir O, Giray S, Arlier Z, et al. Predictors of a good outcome after endovascular stroke treatment with stent retrievers. ScientificWorldJournal 2015;2015:403726 CrossRef Medline

2. Xie Y, Oppenheim C, Guillemin F, et al; THRACE Investigators. Pretreatment lesional volume impacts clinical outcome and thrombectomy efficacy. Ann Neurol 2018;83:178-85 CrossRef Medline

3. Yoo AJ, Chaudhry ZA, Nogueira RG, et al. Infarct volume is a pivotal biomarker after intra-arterial stroke therapy. Stroke 2012;43:1323-30 CrossRef Medline

4. Campbell BC, Donnan GA, Mitchell PJ, et al. Endovascular thrombectomy for stroke: current best practice and future goals. Stroke Vasc Neurol 2016;1:16-22 CrossRef Medline 
5. Albers GW, Marks MP, Kemp S, et al; DEFUSE 3 Investigators. Thrombectomy for stroke at $\mathbf{6}$ to 16 hours with selection by perfusion imaging. N Engl J Med 2018;378:708-18 CrossRef Medline

6. Campbell BC, Mitchell PJ, Kleinig TJ, et al; EXTEND-IA Investigators. Endovascular therapy for ischemic stroke with perfusionimaging selection. $N$ Engl J Med 2015;372:1009-18 CrossRef Medline

7. Yoo AJ, Verduzco LA, Schaefer PW, et al. MRI-based selection for intra-arterial stroke therapy: value of pretreatment diffusionweighted imaging lesion volume in selecting patients with acute stroke who will benefit from early recanalization. Stroke 2009;40: 2046-54 CrossRef Medline

8. Yoo J, Choi JW, Lee SJ, et al. Ischemic diffusion lesion reversal after endovascular treatment. Stroke 2019;50:1504-09 CrossRef Medline

9. Edlow BL, Hurwitz S, Edlow JA. Diagnosis of DWI-negative acute ischemic stroke: a meta-analysis. Neurology 2017;89:256-62 CrossRef Medline

10. Schuleri KH, Centola M, George RT, et al. Characterization of periinfarct zone heterogeneity by contrast-enhanced multidetector computed tomography: a comparison with magnetic resonance imaging. J Am Coll Cardiol 2009;53:1699-1707 CrossRef Medline

11. Nogueira RG, Jadhav AP, Haussen DC, et al; DAWN Trial Investigators. Thrombectomy 6 to 24 hours after stroke with a mismatch between deficit and infarct. N Engl J Med 2018;378:1121 CrossRef Medline

12. Federau C, Gallichan D. Motion-correction enabled ultra-high resolution in-vivo 7T-MRI of the brain. PLoS One 2016;11:e154974 CrossRef Medline

13. Benameur K, Bykowski JL, Luby M, et al. Higher prevalence of cortical lesions observed in patients with acute stroke using high-resolution diffusion-weighted imaging. AJNR Am J Neuroradiol 2006;27:1987-89 Medline

14. Hotter B, Galinovic I, Kunze C, et al. High-resolution diffusionweighted imaging identifies ischemic lesions in a majority of transient ischemic attack patients. Ann Neurol 2019;86:452-57 CrossRef Medline

15. Goyal M, Menon BK, Derdeyn CP. Perfusion imaging in acute ischemic stroke: let us improve the science before changing clinical practice. Radiology 2013;266:16-21 CrossRef Medline

16. Sheth KN, Terry JB, Nogueira RG, et al. Advanced modality imaging evaluation in acute ischemic stroke may lead to delayed endovascular reperfusion therapy without improvement in clinical outcomes. J Neurointerv Surg 2013;(Suppl 1):i62-65 CrossRef Medline

17. Federau C, Christensen S, Mlynash M, et al. Comparison of stroke volume evolution on diffusion-weighted imaging and fluid-attenuated inversion recovery following endovascular thrombectomy. Int J Stroke 2017;12:510-18 CrossRef Medline 